\title{
An Adaptive Packet Scheduling Algorithm in OFDM Systems with Smart Antennas
}

\author{
Zhifeng Diao, Dongxu Shen, Victor O. K. Li \\ Department of Electrical and Electronic Engineering \\ The University of Hong Kong, Hong Kong, China
}

\begin{abstract}
To maximize system throughput and guarantee the quality of service (QoS) of multimedia traffic in Orthogonal Frequency Division Multiplexing (OFDM) systems with smart antennas, a new packet scheduler is introduced to consider QoS requirements, packet location in the frame, and modulation scheme. In OFDM, several consecutive subcarriers are grouped as a frequency subband. Each subband in a frame can be reused by several users with smart antennas. In this paper, based on the Best-Fit algorithm proposed for TDMA and the physical features of OFDM, a new packet scheduler is proposed to allocate different BER-classified traffics into the frame. Adaptive modulation is also applied in the scheduler. When compared with existing schedulers, our scheduler achieves higher system capacity with much reduced complexity. The use of adaptive modulation further enhances the system capacity. Simulation results demonstrate that as the traffic load increases, the new scheduler has much better performance in system throughput, average delay, and packet loss rate.
\end{abstract}

\section{INTRODUCTION}

Spectrum is an important wireless resource, and the scarcity of the spectrum demands high bandwidth efficiency. Space division multiple access (SDMA) allows the reuse of bandwidth by multiplexing users in the same frequency band. SDMA has been applied to TDMA and CDMA systems. In [1, 2], a smart channel assignment algorithm is introduced in SDMA/TDMA systems. In [4], smart antenna is applied to CDMA systems.

Orthogonal Frequency Division Multiplexing (OFDM) is considered as a multiple access scheme for wireless broadband networks. Many papers have treated the resource management problem in OFDM systems. In [5], the whole bandwidth of an OFDM system is divided into several sub-bands. Three schemes are proposed to schedule the bandwidth resource. However, SDMA is not considered. An overview on the dynamic packet assignment for high-efficiency resource management in OFDM systems can be found in [4]. In [2], an algorithm is proposed to allocate spatially separable users in the same subcarrier. However, this paper considers each subcarrier separately, and the scheduler is at the bit level, not the packet level. Thus the complexity is very high.

In multimedia OFDM/SDMA networks, different traffics have different BER requirements. When users with low BER requirements are transmitted together with users having high BER requirements on the same channel, the BER performance exceeds their needs, because the system must satisfy the most stringent BER requirements of all packets. Therefore, the number of users that can be accommodated in the channel is reduced.
In this paper, we consider an OFDM/SDMA system, in which the bandwidth is divided into subbands composed of several consecutive subcarriers. The same subband can be used by several users at the same time. Further, a number of OFDM symbols are grouped as an OFDM frame. We first apply the Random-Fit, First-Fit, and Best-Fit packet allocation schemes proposed in [1] to OFDM/SDMA systems. Then, based on the Best-Fit scheme, we propose a BER-classified Best-Fit packet scheduler. The scheduler classifies all traffic into classes according to the BER requirements, and allocate packets of the same class in the same frequency subband. Adaptive modulation is applied. It is found that the BER-classified Best-Fit scheduler always has the best performance, in terms of system throughput, average delay, and packet loss rate. Adaptive modulation also improves the system performance when combined with the scheduler.

The remainder of this paper is organized as follows. In Section II, we introduce the system architecture. Basic packet scheduling algorithms are given in Section III. Then the BER-based traffic classification is introduced and the Best-Fit scheduler is illustrated in details in Section IV. The simulation results are shown in Section V. Section VI is the conclusion.

\section{SYSTEM MODEL}

In this section, we describe the structure of the mobile terminal and base station. When a terminal has packets to transmit, it sends an admission-request packet through a reservation request slot, from which the base station obtains the spatial signature and traffic information of the terminal. Then the base station assigns subbands to the terminal depending on the QoS request and the traffic information. In this paper, we only consider the scheduling of packets after the terminals are admitted.

\section{A. System Structure}

We consider an OFDM/SDMA system which consists of $N_{u}$ mobile terminals, each equipped with a single antenna. The base station has an $M$-element adaptive linear antenna array, capable of separating $K \leq M$ users. In OFDM systems, the total wireless bandwidth is divided into $N_{c}$ orthogonal subcarriers. In this paper, we group a fixed number of subcarriers into a subband, which is called a frequency subband.

Fig. 1 gives the structure of the base station in an OFDM/SDMA system. We consider the downlink of the system, and as in $[1,4]$, we assume that the base station has 


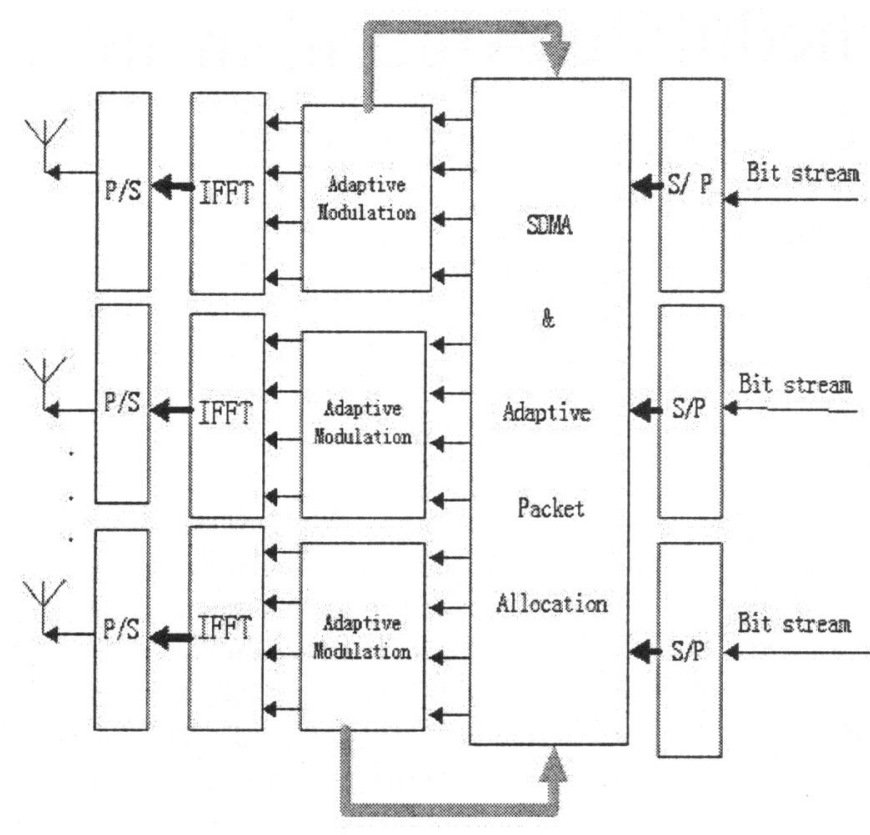

Fig. 1. Base station downlink structure.

perfect user channel information. The scheduler will distribute user packets into the subband. After beamforming, adaptive modulation is applied. These packets are then fed in blocks of symbols into an $N$-tap inverse fast Fourier transform (IFFT) operator to generate the time domain sequence. Power is also adaptively allocated among the users. Then the sequence is transmitted. At the mobile terminal, the signal is converted to a parallel data stream, processed by FFT, and finally demodulated. Superscripts $T$ and $H$ denote the transpose and complex conjugate transpose of a vector or matrix.

\section{B. Beam Forming and Power Allocation}

Let $\mathbf{H}_{k}(n)=\left[H_{1, k}(n), H_{2, k}(n) \ldots, H_{M, k}(n)\right]^{T}$ be the frequency channel response between user $k$ and the $M$ antennas of the base station on subcarrier $n$. Let $\mathbf{H}(n)=$ $\left[\mathbf{H}_{1}(n), \ldots, \mathbf{H}_{K}(n)\right]^{T}$ be the channel matrix between the base station antennas and the $K$ mobile terminals, $\mathbf{X}_{n}=$ $\left[x_{n, 1}, \ldots x_{n, K}\right]^{T}$ be the data at subcarrier $n$ of all users, and $\mathbf{Y}_{n}=\left[Y_{n, 1}, \ldots, Y_{n, K}\right]^{T}$ be the received signal at subcarrier $n$ of all $K$ active mobile terminals.

At the base station, the SDMA module generates a set of weight vectors for each subcarrier of each user. The weight vector can be denoted as $\mathbf{V}_{n, k}=\left[v_{n, k}^{1}, v_{n, k}^{2}, \ldots, v_{n, k}^{M}\right]^{T}$, which is one of the eigenvectors of the channel matrix $(\mathbf{H}(n))^{H}(\mathbf{H}(n))$, and all these eigenvectors are orthogonal to each other [9]. By steering the set of beamforming vectors, the signal of different users on the same subcarriers can be separated at the mobile terminals.

To achieve good system throughput with smart antennas, we need to optimally allocate the power to all users. Let $P_{1}, P_{2}, \ldots, P_{K}$ be the power allocated to each user. The power for each user should satisfy $\sum_{j=1}^{K} P_{j}=P$, where $P$ is a constant corresponding to the power constraint. The multi-user power allocation problem can be transformed into a parallel single user power allocation, as in [9].

The signal received by the $k$ th mobile terminal can be expressed as

$$
\begin{aligned}
\mathbf{Y}_{n, k}= & \left(\mathbf{V}_{n, k}\right)^{H} \sqrt{P_{k}} \mathbf{H}_{k}(n) x_{n, k} \\
& +\sum_{j \neq k}^{K}\left(\mathbf{V}_{n, j}\right)^{H} \sqrt{P_{j}} \mathbf{H}_{k}(n) x_{n, j}+\eta_{n, k}
\end{aligned}
$$

where $\mathbf{H}_{k}(n)$ is the channel vector of subcarrier $n$ between user $k$ and the base station, $x_{n, k}$ and $x_{n, j}$ are the signal for each user. The first term in (1) is the desired signal, the second term is the interference from other users, and $\eta_{n, k}$ is the additive white Gaussian noise with variance $\sigma^{2}$. The signalto-interference and noise ratio $\left(S I N R_{n, k}\right)$ at subcarrier $n$ of user $k$ is

$\frac{P_{k}\left(\mathbf{V}_{n, k}^{H} \mathbf{H}_{k}(n) \mathbf{V}_{n, j} x_{n, k}\right)\left(\mathbf{V}_{n, k}^{H} \mathbf{H}_{k}(n) \mathbf{V}_{n, j} x_{n, k}\right)^{H}}{\sum_{j \neq k}^{K} P_{j}\left(\mathbf{V}_{n, k}^{H} \mathbf{H}_{j}(n) \mathbf{V}_{n, j} x_{n, j}\right)\left(\mathbf{V}_{n, k}^{H} \mathbf{H}_{j}(n) \mathbf{V}_{n, j} x_{n, j}\right)^{H}+\sigma^{2}}$.

In this paper, we consider the wireless channel as slow fading. As several consecutive frequency subchannels are grouped as a subband, the channel gains on the subchannels in a subband have small variations. Then the channel quality can be represented by the average SINR. Let $\overline{S I N R}_{b, k}$ be the average SINR of the $b$ th frequency subband assigned to user $k$. The average SINR value can be given as

$$
\overline{\operatorname{SINR}}_{b, k}=\frac{\sum_{q=q_{s}}^{q_{e}} S I N R_{n, k}}{q_{e}-q_{s}+1}
$$

where $q_{s}$ and $q_{e}$ denote the subcarrier indices of the start and the end of the frequency subband. The average SINR value determines the BER performance.

Next, we discuss the relationship between SINR and the BER performance. We consider a family of M-QAM signal constellations, where $\mathrm{M}$ denotes the number of points in each signal constellation. From [7], we know that the BER of a user with M-QAM modulation is approximated as $B E R \approx$ $0.2 e^{-1.5 \frac{S I N R}{N-1}}$. Then the minimum SINR value to support $B E R \leq p$ for M-QAM modulation is

$$
S I N R_{\text {threshold }}=-\frac{\ln (5 p)}{1.5}(M-1)
$$

\section{BASIC OFDM/SDMA PACKET AlLocation ALGORITHMS}

\section{A. OFDM/SDMA Packet Scheduling}

We consider a scheduler as shown in Fig. 2. All packets will be assigned a priority before being put into a fist-in-firstout (FIFO) queue. Then the packets are allocated into frames. We require a packet to be allocated in the subband of a frame once the SINR requirements of all packets in the subband are satisfied. In TDMA systems with smart antennas [8], several schemes are proposed to dynamically allocate time slots to different users. Such schemes can be extended to OFDM systems. These algorithms are described in an increasing order of complexity as Random-Fit, First-Fit and Best-Fit.

All stations are numbered from the set $\{1,2, \ldots, K\}$, and each station can be referred by its ID. $\xi(i)$ is defined to be the 


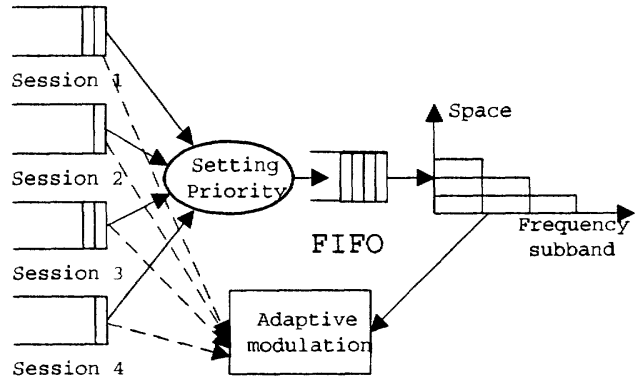

Fig. 2. Description of scheduler.

set of stations currently allocated in frequency slot $i$. Let $\chi$ be the set of mobile terminals with unallocated packets, and $S I N R_{\text {threshold }}$ be the SINR threshold value to guarantee the BER performance.

\section{B. Basic Scheduling Algorithms}

The Random-Fit algorithm is very simple and works as follows. The system randomly picks a terminal from the set $\chi$. Suppose a packet has been put into the frequency subband $d$. Then the scheduler will check each packet in the set $\xi(d)$ to see if the SINR value is above the desired threshold. The FirstFit algorithm is applied next. For each frequency subband, the system will check all packets of the terminals in set $\chi$ to see if they can be assigned in the frequency subband. Once a suitable subband is found, the packet is allocated to the subband. Finally the Best-Fit scheme is applied. Since the base station has perfect channel knowledge of all terminals, the scheduler is able to predict the received power at the receiver terminal if the packet is transmitted in the frequency subband. Due to changes in wireless channel conditions, the received power in each subband is different. From (3), we can get $\overline{S I N R_{b}}$ for the bth frequency subband. The $S I N R$ margin value for each subband is calculated as $S I N R_{\text {margin }, b}=\overline{S I N R_{b}}-S I N R_{\text {threshold }}$. We pick a subband $b$ with the largest SINR margin value. We then check whether the SINR requirements of the packets in $\xi(b)$ can be satisfied if the packet is allocated in the subband. If not, we try the next subband.

\section{BER-CLASSIFIED PACKET SCHEDULING AND ADAPTIVE MODULATION ALGORITHM}

Wireless networks will serve a mixture of voice, data, and video traffic. Each service has its special QoS requirements, such as maximum tolerable BER and timeout requirements. When multimedia traffic is transmitted in OFDM/SDMA systems, the system capacity is largely limited by the traffic with the highest BER requirement.

The main objectives of our scheduler are to maximize the throughput and to minimize the packet error rate. In this algorithm, traffic is classified by BER requirements. Then packets of the same class are allocated in the same frequency subband. In this way bandwidth can be used efficiently. Consider that each traffic class $C_{q}$, for $q=1,2 \ldots, T$, has a BER specification given by $B\left(C_{q}\right)$. BER is only determined by SINR given FEC and modulation.
These objectives can be achieved by the scheduler with two steps. The first is packet priority determination and the second is packet allocation in the frame. The two steps are discribed as follows.

\section{A. Packet Priority Determination}

Many papers have proposed methods to determine packet priority, such as [8]. The computation of packet priority is done dynamically at the start of each frame. In this paper, we consider the case that each mobile terminal only supports one type of traffic.

We assume the buffer of terminal $k$ has $L_{k}$ packets, whose deadlines are $t_{1}, t_{2}, \ldots t_{L_{k}}$. Let the current time be $t_{c}$. Then for the $i$ th packet in the buffer, the minimum transmit rate is $r_{i}=$ $\frac{1}{t_{c}-t_{i}}$. The total transmission rate at the current frame should be $\sum_{i=1}^{L_{k}} \frac{1}{t_{c}-t_{i}}$, which indicates how many frequency subbands should be allocated in the frame for terminal $k$. Based on this idea, we define the priority of each packet in the queue as

$$
P_{k}(i)=\sum_{j=i}^{L_{k}} \frac{1}{t_{c}-t_{j}} .
$$

This priority definition is based on the total transmission rate of the packet and the remaining packets backlogged. This priority reflects the required transmission rate of the terminal. The longer the queues, the larger the priority. Though the priority definition is based on heuristics, it works well as shown in the simulations.

\section{B. BER-Classified Packet Allocation}

The scheduler also keeps track of packets in subbands. For each packet, the scheduler needs two parameters. The first is an $I D$ to identify the mobile terminal. In an OFDM/SDMA frame, only one packet of a mobile can be transmitted in the same frequency subband. The second parameter is the traffic class $C_{q}$. It is used for BER scheduling. The packet allocator will attempt to arrange the packets in the following steps:

STEP 1. Search the subbands that contain the same traffic class $C_{q}$. If a set of such subbands are found, the scheduler attempts to insert the packet into the frequency subband which has the largest $S I N R$ margin value. Then it checks whether the $S I N R$ requirements of all the packets in the subband can be satisfied. If yes, the packet is allocated in the subband. If not, the subband with the second largest $S I N R$ margin is selected. If the packet can not be allocated when all subbands with traffic class $C_{q}$ are tried, go to Step 2 .

STEP 2. Search an empty subband. If found, arrange the packet in the empty subband. If no empty subband is found, the packet scheduler proceeds to Step 3 .

STEP 3. Search the frequency subband that has packets with more stringent BER requirements. In other words, the scheduler will search for a frequency subband with traffic class $C_{q-1}$, which has more stringent BER requirements than $C_{q}$. If such subbands are found, the scheduler will try to place the packet into the frequency subband by the Best-Fit algorithm. If the subbands can not accommodate the packet, the scheduler checks subbands of traffic classes $C_{q-2}, \ldots, C_{1}$, 
when the packet is allocated. If the packet still can not be allocated, go to Step 4.

STEP 4. Search the frequency subband that has packets with more relaxed BER requirements. The scheduler looks for a frequency subband with traffic class $C_{q+1}$. If such subbands are found, based on the Best-Fit algorithm the scheduler will test whether the packet can be added into the subband. Then the packets in this subband are converted into class $C_{q}$, since more stringent BER requirement in the subband must be satisfied. Similarly, the scheduler looks for subbands with traffic classes $C_{q+1}, C_{q+2}, \ldots, C_{T}$. This operation will stop until the last subband with traffic class $C_{T}$ is reached.

\section{Adaptive Modulation}

In this algorithm, we consider a family of M-QAM signal constellations of BPSK, QPSK, and 16-QAM. All packets have the same fixed length. BER performance is related to both SINR and modulation. A high SINR value in a frequency subband enables the utilization of high M-QAM modulation level, which increases the system throughput.

After the above four steps of the scheduler operation, we consider adaptive modulation for users who still have packets in the buffer waiting for transmission. First, we should identify the frequency subband that contains the packets of these users. Second, we increase the modulation level of these packets. Since the packet length is fixed, if we increase the modulation level by one step, the number of bits that a subband can accommodate doubles, and two packets of the same user can be merged as one. Then the scheduler will check that frequency subband to determine if the SINR of all packets can be satisfied. If yes, the packet modulation level is increased. Otherwise, find the next frequency subband that contains the packet of that user. This operation will stop when all the frequency subbands are considered or there are no packets in the queues.

\section{Simulation Results with Multimedia TrafFiC}

In this section, we present the simulation results for multimedia traffic. The packet scheduling algorithms include the Random-Fit, First-Fit, Best-Fit, and BER-Classified Best-Fit. Adaptive modulation is combined with the BER-Classified Best-Fit algorithm.

\section{A. Simulation Setup}

The base station has four antennas serving 100 mobile users. In OFDM, the bandwidth is divided into 64 subcarriers. Only 48 subcarriers are used to transport data packets, which are divided into 8 frequency subband. In the other words, 6 subcarriers in a frame are grouped as a frequency subband. We group 1000 OFDM symbols as a frame, which lasts for $4 \mathrm{~ms}$. All packets have the same fixed length. The channel response during one frame time is regarded as a constant. The base station has perfect channel information for each user.
TABLE I

MULTIMEDIA QOS REQUIREMENTS

\begin{tabular}{|c|c|c|c|c|}
\hline $\begin{array}{c}\text { Traffic } \\
\text { Type }\end{array}$ & BER & Modulation & $\operatorname{SINR}(\mathrm{dB})$ & $\begin{array}{l}\text { Time } \\
\text { Out (ms }\end{array}$ \\
\hline \multirow{2}{*}{ Voice } & \multirow{2}{*}{$10^{-3}$} & BPSK & 3 & \multirow{2}{*}{6} \\
\hline & & QPSK & 10 & \\
\hline \multirow{2}{*}{$\begin{array}{c}\text { CBR } \\
\text { Digital } \\
\text { Audio } \\
\end{array}$} & \multirow{2}{*}{$10^{-4}$} & BPSK & 5 & \multirow{2}{*}{25} \\
\hline & & QPSK & 15 & \\
\hline \multirow{2}{*}{$\begin{array}{c}\text { CBR } \\
\text { Video }\end{array}$} & \multirow{2}{*}{$10^{-5}$} & BPSK & 6 & \multirow{2}{*}{15} \\
\hline & & QPSK & 18 & \\
\hline \multirow{2}{*}{$\begin{array}{l}\text { VBR } \\
\text { Video }\end{array}$} & \multirow{2}{*}{$10^{-6}$} & BPSK & 7 & \multirow{2}{*}{15} \\
\hline & & QPSK & 21 & \\
\hline $\begin{array}{c}\text { Computer } \\
\text { Data }\end{array}$ & $10^{-7}$ & BPSK & 8 & 200 \\
\hline
\end{tabular}

1) Multimedia Traffic Models: Voice traffic model: The speech source creates a pattern of talk spurts and gaps. In our simulation, the model is based on the three-state Markov model presented in [6]. During the spurt states, we assume the mobile model generates a data rate of $16 \mathrm{~kb} / \mathrm{s}$.

CBR Digital Audio Traffic: This model represents the production of continuous bit stream of digital FM Stereo Audio. The average holding time of an audio call is $360 \mathrm{~s}$ with an exponential distribution .

CBR Video Traffic: In this model, a continuous bit stream is generated at $220 \mathrm{~kb} / \mathrm{s}$. The interval between two packet transmissions is $0.05 \mathrm{~s}$.

VBR Video Traffic: The video traffic is modeled by an 8state Markov-modulated Poisson Process (MMPP). In each state, the packet arrival satisfies a Poisson process. The average duration in each state is set to be $30 \mathrm{~ms}$. The average bit rate is the same as in CBR video traffic, but BER threshold and delay requirements are different.

Computer data traffic: The transmission interval is exponentially distributed and the mean bit rate is $30 \mathrm{~kb} / \mathrm{s}$.

The QoS requirements of these traffics are listed in Table I. To simplify the simulation complexity, only BPSK and QPSK modulations are considered. By (3), the SINR threshold values with different modulations can be calculated by the BER requirements of different traffic classes. The timeout values of traffic classes are also provided in Table I.

2) Numerical Results: In this section, we give the simulation results with multimedia traffic. The new mobile terminal arrival rates for different traffic classes are maintained constant throughout the simulations. The voice traffic is $50 \%$ of the total system traffic, computer data is $20 \%$, and $10 \%$ for each of the other three traffic types.

Fig. 4 gives the system throughput of the schedulers of Best-Fit, BER-Classified-Best-Fit, and BER-Classified-BestFit with adaptive modulation. At light cell load, the system throughputs of all schedulers are the same. As cell load increases, the performance gap becomes wider. It is obvious that BER-Classified algorithm is better than Best-Fit. Adaptive modulation also contributes to the system throughput.

In Fig. 5, the average packet loss rates of different schedulers are compared. The simulation results show that the average packet loss rate of Best-Fit is always larger than the other two schedulers. Adaptive modulation with Best-Fit also 


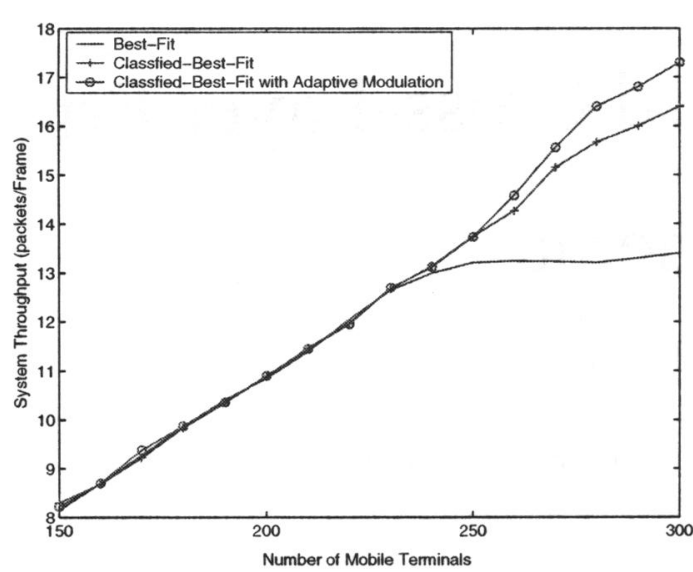

Fig. 3. Multimedia system throughput comparison.

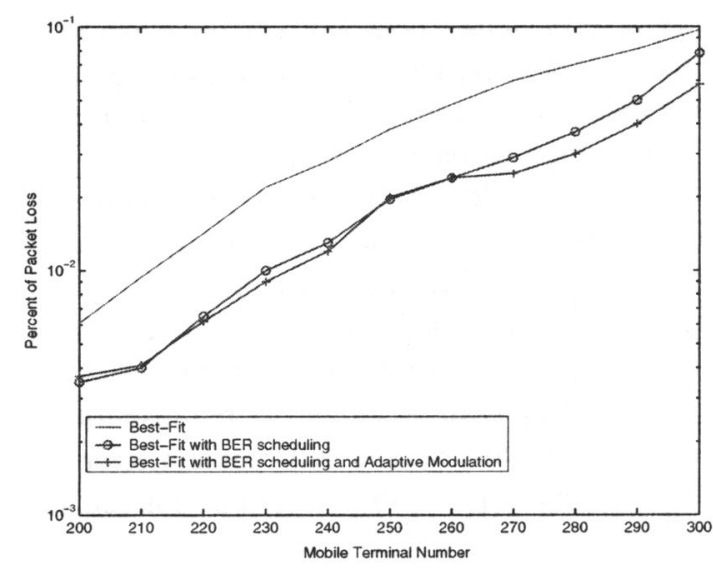

Fig. 4. Packet loss rate comparison.

reduces the packet loss rate.

The average packet delay performance of three packet schedulers are shown in Fig. 6. In the simulation, it is found that the delay performance is related to the packet loss rate. In order to fairly compare the delay performance of different schedulers, when we evaluate the average delay performance, the lost packets are also included, and the time delay is set to be the same as the timeout value. We find that the delay of the Best-Fit scheduler is always larger than other schedulers. BER-Classified Best-Fit scheduler with adaptive modulation also reduces the average packet delay.

\section{Conclusions}

In this paper, we propose an adaptive packet allocation scheme with BER scheduling for OFDM/SDMA systems. All traffics are classified by the BER requirement. The algorithm tries to allocate packets with the same BER class in a frequency subband. In this way, the system throughput is improved, and the complexity is also reduced when compared with the Best-Fit algorithm. We simulate multimedia traffic with different QoS requirements. In terms of the throughput, delay, and packet loss rate, the BER-Classified Best-Fit scheduler is always better than the Best-Fit scheduler. Further adaptive modulation always enhances system performance.

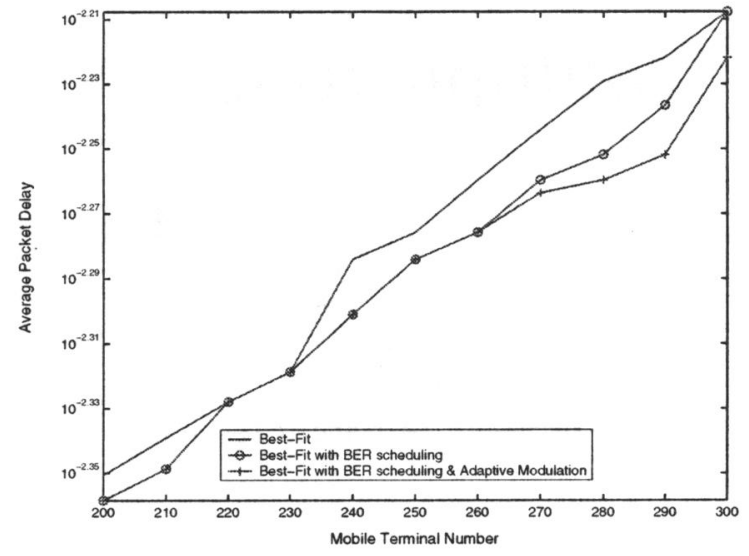

Fig. 5. Packet delay comparison

\section{ACKNOWLEDGMENT}

This research is supported in part by the Research Grants Council of the Hong Kong Special Administrative Region, China (Project No. HKU 7047/00E).

\section{REFERENCES}

[1] F. Shad, T. D. Todd, V. Kezys and J. Litva, "Dynamic slot allocation (DSA) in indoor SDMA/TDMA using a smart antenna basestation," IEEE/ACM Trans. Networkoing, vol. 9, no. 1, pp. 69 -81, Feb. 2001.

[2] F. Piolini and A. Rolando, "Smart Channel-Assignment Algorithm for SDMA Systems," IEEE Trans. Microwave And Tech, vol. 47, No. 6, pp. 693-699, Jun. 1999.

[3] J. C. Liberti and T. S. Rappaport, Smart Antennas for Wireless Communications: IS-95 and third Generation CDMA Applications. Englewood Cliffs, NJ: Prentice Hall, 1999.

[4] W. Anchun, X. Liang, Z. Shidong, X. Xibin, and Y. Yan, “Dynamic Resource management in the fourth generation wireless systems," ICCT2003, vol. 2, pp. 9-11, Beijin. China, Apr. 2003.

[5] J. Chuang, L. J. Cimini, et al., "High-speed Wireless Data Access Based on Combining EDGE with Wideband OFDM," IEEE Comm. Magazine, vol. 37, pp. 92-97, Nov. 1999.

[6] D. J. Goodman and S. X. Wei, "Efficiency of packet reservation multiple access," IEEE Trans. Ve. Technol., vol. 40, pp. 170-176, Feb. 1991.

[7] A. J. Goldsmith and S. -G. Chua, " Variable-rate variable-power MQAM for fading channels," IEEE Trans. Commun., vol. 45, No. 10, pp. 1218-1230, Oct. 1997

[8] H. Yin, and H. Liu, "Performance of Space-Division Multiple- Access (SDMA) with scheduling," IEEE Trans On Wireless Comm, vol. 1, No. 4, pp. 611-618, Oct. 2002.

[9] S. Duan, Y. Xu and W. Song, "Power Allocation for MIMO-OFDM Systems with Multi-user Decoupling and Scheduling," PIMRC, vol. 1, pp. 911-915, Sep. 2003. 